\title{
Wireless Fluorimeter for Mobile and Low Cost Chemical Sensing: A Paper Based Chloride Assay
}

\author{
Petar Kassal $^{\mathrm{a}}$, Matthew D. Steinberg ${ }^{\mathrm{b}}$, Ema Horak ${ }^{\mathrm{c}}$, Ivana Murković Steinberg ${ }^{\mathrm{a}, *}$
}

${ }^{a}$ Faculty of Chemical Engineering \& Technology, University of Zagreb, Marulićev trg 19, HR-10000 Zagreb, Croatia

${ }^{\mathrm{b}}$ GoSense Wireless Ltd, 57A Moorfield Road, Duxford, Cambridge, CB22 4PP, UK

${ }^{c}$ Computational Organic Chemistry and Biochemistry Group, Ruđer Bošković Institute, Bijenička cesta 54, HR-10000 Zagreb, Croatia

${ }^{*}$ Corresponding author:

Tel : +385 (0)1 4597287

E-mail: ivana.murkovic@fkit.hr

\begin{abstract}
Wireless chemical sensors are increasingly finding use as analytical devices in healthcare diagnostics, wearable sensing (sweat analysis) and in the rapidly emerging area of the Sensor Internet of Things (SloT). In such wireless scenarios, the application of fluorescencebased sensors is lagging behind other types of transduction mechanisms. In this work a new low-cost and highly portable wireless fluorimeter for optical chemical fluorescence intensity measurements is presented (bill-of-materials approximately €20). The fluorimeter is programmed and communicates wirelessly with mobile devices or personal computers by radio-frequency identification (RFID) or near-field communication (NFC). This novel mobile analytical system is highly adaptable for use with different fluorescent sensor chemistries and analytes. The fluorimeter has been evaluated in the laboratory in two sets of proof-ofconcept experiments: firstly fluorimetry in solution, where the fluorescence intensities of fluorescein were recorded, and secondly chloride sensing via a paper-immobilised quinine sulphate fluorescent indicator. The latter represents the first demonstration of wireless (radio-frequency) fluorescence-based chloride sensing. Satisfactory analytical performance was achieved in the entire range of sweat chloride concentrations. The wireless fluorimeter system presented here could make a significant contribution to several emerging areas of
\end{abstract}


mobile chemical sensor research, including wearable sensors for healthcare and sport, mobile point-of-sample diagnostics as well as more generally for the Sensor Internet of Things.

\section{Keywords}

Wireless chemical sensor; Fluorescent sensor; Radio-frequency identification; Paper-based sensor; Chloride sensor; Wearable sensor

\section{Introduction}

Wireless chemical sensors (WCS) are analytical devices that combine chemical sensing capability with integrated wireless data transfer. They are increasingly being leveraged in healthcare diagnostics, homeland defence, as well as more generally in the rapidly emerging area of the Sensor Internet of Things (SloT). Proponents of the Internet of Things (IoT) predict that there will soon be a vast number of connected devices, including chemical sensors that are able to monitor and sense their ambient environment and to share this data with the internet and cloud-based computing services. This has clear implications for homeland security, environmental monitoring, food and chemical supply-chain logistics, and provision of home-based healthcare services [1-3].

The various advantages of radio-frequency identification (RFID) and near field communication (NFC) as a mobile communications technology have been discussed in detail [3-5]. In brief, RFID is a low-cost, low-power and short-range transponder technology that will play a significant role in the Internet of Things $[5,6]$. It has been adopted by major industry sectors (food, pharmaceutical, oil \& gas) for asset tracking and allows for scalable connection of individual assets or devices into larger networks. The inclusion of the highfrequency ISO15693 RFID protocol into the NFC specification means that Android-enabled mobile devices such as smartphones and tablets that support NFC can communicate and exchange data with any ISO15693 RFID device. 

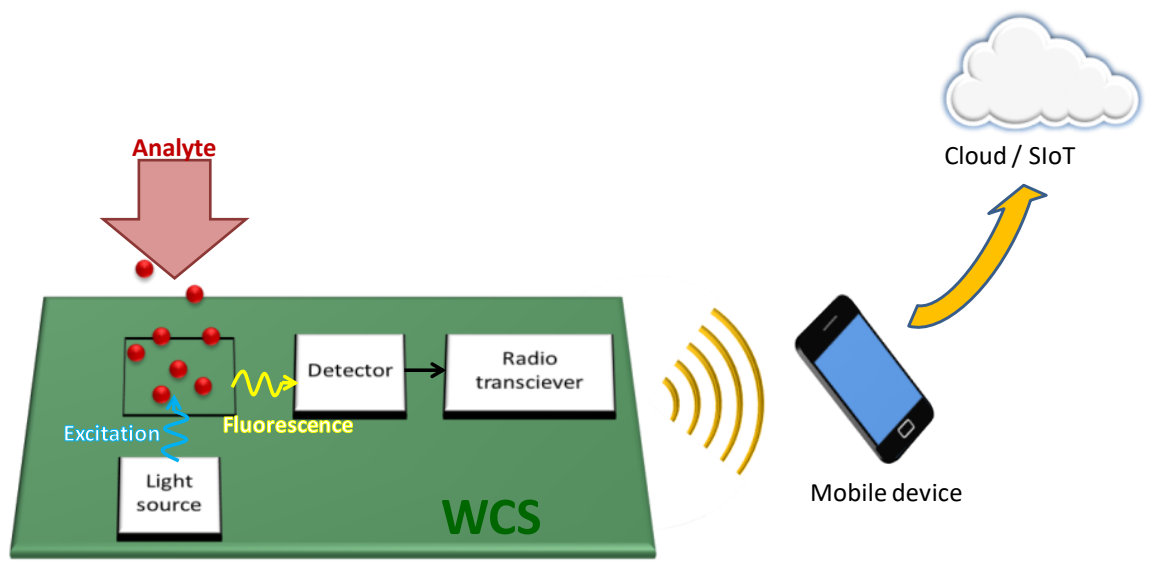

Figure 1. General schematic showing operation of a fluorescence-based wireless chemical sensor.

While optical (bio)chemical sensing offers several advantages over other transduction modes, it is less often used in stand-alone wireless systems due to the large power consumption of light sources [7]. This has been somewhat overcome by using low-power and low-cost optoelectronic components such as light emitting diodes (LEDs) and photodiodes (PDs), which are readily available with optical characteristics that cover the spectral range from ultraviolet to near-infrared [8]. Light emitting diodes and PDs therefore now form the basis of many low-cost optical chemical sensor devices, both colorimetric and fluorescence-based [9-12]. Fluorescent sensors are especially advantageous since they can provide high sensitivity and selectivity, as well as rapid response times [13]. Our research group has demonstrated the use of wireless RFID/NFC-based systems for optical chemical sensing with low-cost optoelectronic components (LEDs, PDs): the system was optimised for operation in transmission [14] and reflectance [15] modes. However, fluorescence measurements with low-cost optoelectronics are much more challenging, especially without additional optical components, such as filters, lenses etc. The main reason for this is the relatively weak fluorescence signal and interference from the high-intensity excitation light. Nevertheless, wireless chemical sensors based on fluorescence measurements, utilising LEDs and PDs, have also been demonstrated in the literature. For example, an RFID/NFCbased sensor was developed for the determination of gaseous oxygen in food packaging by ultra-violet (UV) LED-excited luminescence of an $\mathrm{O}_{2}$ sensing membrane [16, 17]. An NFCbased implantable glucose sensor, relying on fluorescence intensity changes of a glucose indicating hydrogel has been developed [18], as well as a swallowable wireless capsule which uses an LED and a PD to detect the presence of a fluorescent dye in the gastrointestinal (Gl) tract as a means of monitoring Gl bleeding [19]. 
Smartphones also provide a versatile platform for the development of optical sensors and biosensors, but achievement of satisfactory analytical performance using integrated cameras as the detector component is often challenging. An example of successful smartphone camera utilisation is smartphone-based spectroscopy, which can easily replace expensive and bulky laboratory spectrometers [20].

One of the major application groups of wireless chemical sensors - wearable chemical sensors - have seen immense development in the last several years, owing to the abundance of (bio)chemical information which can be extracted from sweat or other body fluids by non-invasive means [21-25][7]. For instance, chloride determination in sweat is a gold standard for diagnosing cystic fibrosis $[26,27]$ and wearable sensors for sweat chloride utilising electrochemical transduction [28, 29] and colourimetric measurements [30] have already been developed. However, wireless or wearable fluorescent chloride sensors have not been developed to our knowledge.

In this work a new low-cost, highly mobile and versatile RFID/NFC wireless fluorimeter is presented, designed for use with solution-based or immobilised fluorescent indicators. The key feature of this device is the ability to communicate wirelessly via near-field communication (NFC) or radio-frequency identification (RFID) with mobile phones and tablets, Fig.1. Major drawbacks of analytical platforms that use the integrated smartphone camera as the optical detector can include interference of ambient light, differences between smartphone cameras and semi-quantitative analyses, all of which are avoided with the independent hardware in this fluorimeter. It is important to mention that differences between smartphones and their integrated cameras and the hardware and low-level software used to control them are highly significant. Our hardware is self-contained and is therefore independent of the smartphone system and manufacturer.

The mobile fluorimeter has been designed, built and subsequently evaluated in a laboratory setting using common fluorophores (fluorescein and quinine sulphate) as model chemical indicators. The two types of proof-of-concept experiments were used for evaluation of the system. In the first experimental set-up, fluorimetry in solution was performed simulating operation of a conventional laboratory spectrofluorimeter, albeit with an instrument of a much smaller size and lower cost, and with wireless data transmission capability. The second application demonstrates a fluorimetric paper test strip which included development of a paper based chloride assay using fluorescence quenching. This demonstrates for the first time fluorescence-based wireless chloride sensing with potential applicability in a wearable sweat sensing scenario. 


\section{Materials and methods}

\subsection{Chemicals and reagents}

Fluorescein solutions in the concentration range $1 \times 10^{-7}$ to $1 \times 10^{-4} \mathrm{~mol} \mathrm{dm}^{-3}$ were prepared by dissolving fluorescein (Sigma-Aldrich, St. Louis, MO, USA) in $0.1 \mathrm{~mol} \mathrm{dm}^{-3} \mathrm{NaOH}$ (Kemika d.d., Zagreb, Croatia). Milli-Q water was used for the preparation of all aqueous solutions. Universal buffer solutions, used in the $\mathrm{pH}$ sensing experiments, with $\mathrm{pH}$ in the range $5.56-$ 8.82 were prepared from citric acid $(0.33 \mathrm{M})$, phosphoric acid $(0.33 \mathrm{M})$, boric acid, sodium hydroxide and hydrochloric acid (all from Kemika). A fluorescein stock solution (c $=1 \times 10^{-3}$ $\mathrm{mol} \mathrm{dm}^{-3}$ ) was prepared in ethanol (Kemika) and subsequently diluted with the prepared buffer solutions. The concentration of fluorescein in the prepared solutions was $6.66 \times 10^{-6}$ $\mathrm{M}$ and the volume percent of ethanol was $0.66 \%$. A $10^{-2} \mathrm{~mol} \mathrm{dm}^{-3}$ quinine sulphate (QS) solution was prepared by dissolving quinine sulphate (Sigma-Aldrich) in $0.05 \mathrm{~mol} \mathrm{dm}^{-3}$ $\mathrm{H}_{2} \mathrm{SO}_{4}$ (Carlo Erba Reagenti, Arese, Italy). The filter paper substrate (FN1) used for chloride sensing was from Ahlstrom Munktell (Stockholm, Sweden). Chloride solutions in the concentration range $1 \mathrm{mM}-100 \mathrm{mM}$ were prepared by dissolving solid $\mathrm{NaCl}$ (Kemika) in Milli-Q water.

\subsection{Wireless fluorimeter design}

The wireless fluorimeter is a small, lightweight and fully programmable autonomous mobile instrument designed for making optical fluorescence measurements. The fluorimeter consists of an excitation LED and a photodiode (PD), which can be arranged in several ways, depending on the desired application: orthogonally around a sample cuvette on separate daughter boards that sit upright along the sides of the cuvette walls (Fig. 2), or soldered directly onto the main board of the fluorimeter as outlined in Fig. 3a The fluorimeter is powered from a standard $3 \mathrm{~V}$ lithium coin cell and communicates with mobile devices via a set of custom ISO15693 RFID/NFC commands. The electronic core of the device is a commercial integrated circuit (MLX90129, Melexis BV, Belgium) which provides the wireless communication functions (RFID and NFC), as well as a programmable data logger function. The same circuit also controls the LED and PD during sample acquisitions, so there is no microprocessor. The main components and wireless communication of the fluorimeter are shown in Figure S1 in the Supplementary Materials. This keeps the cost of the fluorimeter extremely low. In addition to the RFID core there is a $1 \mathrm{Mb}$ memory adequate for storage of 32000 measurements, a constant current source to drive the LED light source, a 
transimpedance amplifier for the photodetector, and a boost regulator to generate a $5 \mathrm{~V}$ power supply from the 3V battery. The MLX90129 contains a true-differential input 16-bit analogue to digital converter. The overall fluorimeter dimensions are $60 \mathrm{~mm} \times 60 \mathrm{~mm} \times 10$ $\mathrm{mm}$. Electronic circuits for the wireless fluorimeter were designed using a schematic capture design and printed circuit board (PCB) layout suite with autorouter (EASY-PC Professional v14.0 with Tracerouter, Number One Systems Ltd., Gloucester, UK). Printed circuit boards for the fluorimeter and the optoelectronic daughter boards were fabricated from standard FR4 materials and populated with surface mount components by automated pick and place followed by reflow solder (L-Tek Elektronika d.o.o., Šentjernej, Slovenia). The per unit bill-ofmaterials cost for the fluorimeter is approximately $€ 20$.

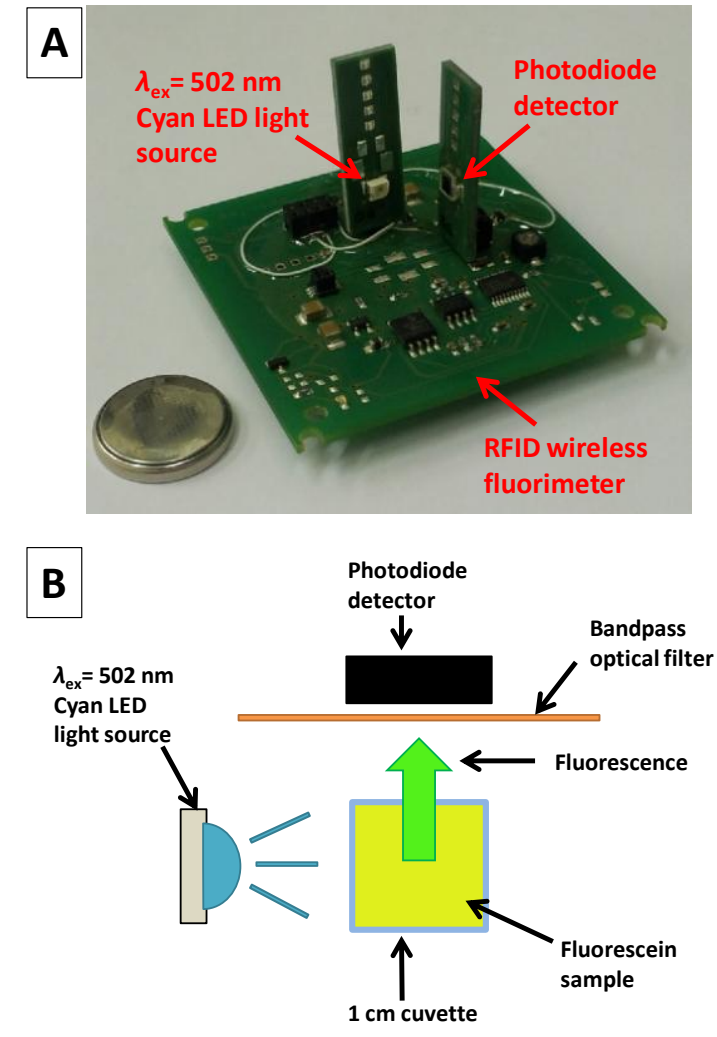

Figure 2. a) Photograph of the RFID/NFC wireless fluorimeter with a CR 2032 coin cell for size reference; b) Diagram of the experimental setup for the fluorimetry in solution experiments

The general protocol for fluorescence intensity measurements with the wireless fluorimeter is given in the Supplementary information. The recorded data is transferred wirelessly to a personal computer running a graphical user dashboard (DVK90129 sensor kit dashboard, Proxima RF Inc, USA) using a USB-powered desktop RFID reader (Proxima RF Inc, USA), or to a smartphone running a custom-designed Android application via NFC. 


\subsection{Methods}

\subsubsection{Fluorimetry in solution}

Fluorescence spectra were recorded on a Varian Cary Eclipse fluorescence spectrophotometer at $25{ }^{\circ} \mathrm{C}$ using $1 \mathrm{~cm}$ path quartz cells. The excitation wavelengths were determined from absorbance maxima. Emission spectra were recorded from $300 \mathrm{~nm}$ to 700 $\mathrm{nm}$ and corrected for the effects of time- and wavelength-dependent light-source fluctuations using a standard of rhodamine 101, a diffuser provided with the fluorimeter and the software supplied with the instrument.

In the wireless experiments, the sample cuvette sits in the centre of the fluorimeter, and the LED and PD are arranged orthogonally around the cuvette on separate daughter boards that sit upright along the sides of the cuvette walls, Fig. 2. Daughter boards are fully interchangeable, thus allowing different LEDs or PDs to be deployed according to the sample assay in use. The optoelectronic components used were a cyan LED light source type HSMK-A100-S00J1, Avago Technologies Ltd., USA (peak wavelength at $502 \mathrm{~nm}$ ), and a broadband photodiode type BPW34S, Osram Opto Semiconductors GmbH, Germany. Due to the broadband response of the employed photodiode, a bandpass optical filter was used in these experiments, to filter out the excitation LED light. The detailed settings of the wireless fluorimeter in these experiments are given in the Supplementary information.

\subsubsection{Paper-based chloride sensing}

Paper test strips were prepared by cutting the filter paper $(0.19 \mathrm{~mm}$ thickness) into $1 \mathrm{~cm} \times 1$ cm square pieces. $20 \mu \mathrm{L}$ of the QS solution was drop cast onto each piece of paper and left to dry overnight. At the start of every experiment, $10 \mu \mathrm{L}$ of the sample chloride solution (1 $\mathrm{mM}-100 \mathrm{mM}$ ) was cast onto the paper test strip. The paper strips were interrogated with the wireless fluorimeter via an optoelectronic probe, which was connected to the wireless fluorimeter with ribbon cable (Fig. 3a). The optoelectronic probe (Fig. 3b) consists of two surface mount optoelectronic components: an excitation LED (OCU-400 UB355, OSA Opto light $\mathrm{GmbH}$, Berlin, Germany), and a narrowband detector photodiode (EPD-470, EPIGAP Optoelektronik $\mathrm{GmbH}$, Berlin, Germany), arranged in a planar configuration on a $3 \mathrm{~cm} \times 1$ cm FR4 daughterboard. Another narrowband diode (EPD-525), with different spectral sensitivity, was also tested. The entire probe is housed within an $8 \mathrm{~mm}$ high 3D-printed ABS plastic holder which blocks the ambient light and regulates the distance between the paper strip and optoelectronics. The settings of the wireless fluorimeter in chloride sensing experiments are given in the Supplementary information. 

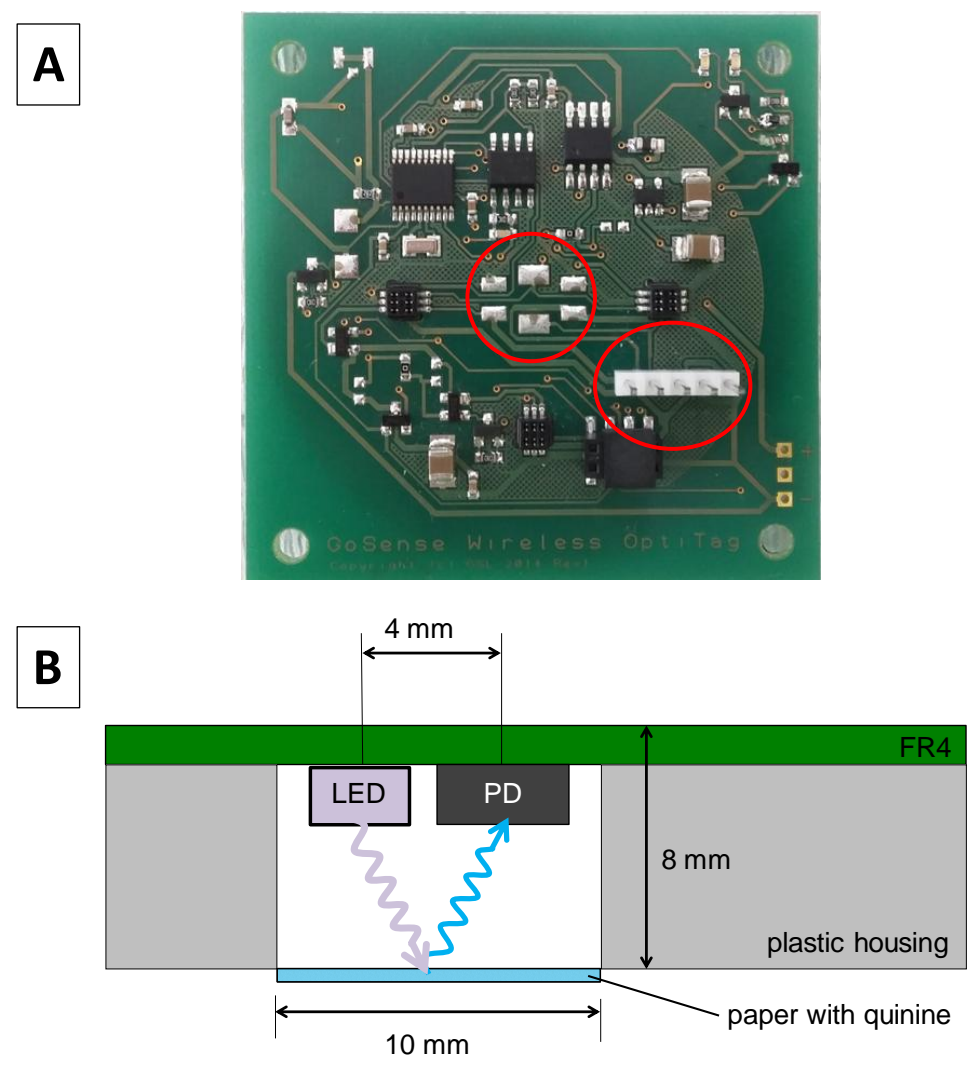

Figure 3. a) Wireless fluorimeter with the solder pads for direct placement of the optoelectronic components outlined, as well as the connector for the optoelectronic probe; b) Cross-section of the optoelectronic probe used in the chloride-sensing experiments demonstrating the arrangement of the optical components.

\section{Results and discussion}

\subsection{Wireless fluorimeter system}

The general system is shown schematically in Figure 1. The key functional blocks within the fluorimeter are the RFID processor (which enables radio communication with a mobile device), the optoelectronic sensor interface for the LED and PD (light source and detector), and the sample cell interface, Figs. 2 \& 3 . The data logger in the fluorimeter is programmed via the PC dashboard to control the optoelectronic interface and data acquisition with the protocol as described above in Materials and Methods.

The optoelectronic components of the fluorimeter comprising the miniature LED source to excite the fluorophore and the photodiode detector to collect the emitted fluorescence signal are highly adaptable for different assays. Both emitter and detector can be changed out 
depending on the chemical fluorescent indicator system used. Furthermore, the optoelectronic components (the LED and the PD) can be mounted on separate orthogonal daughter boards for flexible orientation in any one of three different fixed positions, which allows versatile and flexible measurements with a standard $1 \mathrm{~cm}$ laboratory cuvette. The daughter boards can be placed opposite one another, enabling absorption measurements, or at a $90^{\circ}$ angle (orthogonal) for fluorescence measurements, Fig. 2a. Alternatively, the optoelectronic components can be assembled by soldering directly to the main board of the fluorimeter, which would enable a highly integrated planar $60 \times 60 \mathrm{~mm}$ chemical sensor, Figure 3a. The experimental versatility of the new fluorimeter system is one of the key results of this work.

Notably, the wireless fluorimeter allows for sequential data acquisition from 3 separate inputs. These inputs can come from the PD or from an integral temperature sensor in the MLX90129, or any combination thereof, which brings multiple benefits. For example, the 3 channels can be used to measure the signal from 2 different LEDs (for dual wavelength referencing) as we have previously described [6], or from the excitation LED and from ambient background light as we have done here, or from the integral temperature sensor (for temperature compensation) or indeed any combination of these 3 inputs. In this particular work, only the fluorescence intensity signal and the background light level were recorded.

\subsection{Choice of model experiments}

The analytical performance of the wireless fluorimeter was evaluated through two different types of proof-of-concept experiments: fluorimetry in solution and paper-based chloride sensing. In the solution-based experiments, the wireless fluorimeter was used for determination of different concentrations of fluorescein in solution. Fluorescein is a commonly used luminescence standard with a large quantum yield, it was selected as a model analytical indicator for this work because of its excellent optical properties and availability. All solution-based fluorescence measurements were performed with standard 1 $\mathrm{cm}$ cuvettes, thus simulating operation of conventional laboratory spectrofluorimeters, albeit with an instrument of a much smaller size and lower cost, and with wireless data transmission.

The experimental versatility of the wireless fluorimeter was tested in the next set of experiments in a more typical chemical sensor arrangement: the fluorimeter was used with paper test strips and quinine sulphate (QS) to determine different concentrations of chloride 
via collisional fluorescence quenching. QS was chosen because of its large Stokes shift, which enabled fluorescence measurements without optical filters. Furthermore, QS is readily available and relatively inexpensive (compared to commercial fluorescent chloride probes). These experiments demonstrate compatibility with immobilised fluorophores and feasibility of planar measurements with minimum optical components - just a light source and detector. Furthermore, they demonstrate potential application in wearable sweat chloride sensing. Wearable chloride sensing has been explored with electrochemical sensors, both for exercise monitoring [29] or as a means of diagnosing cystic fibrosis [28]. Determination of chloride in sweat with fluorescent sensors has also been demonstrated, but these systems lacked wireless communication and relied on discrete analysis of collected sweat with standard expensive laboratory equipment [31] or custom instruments [32].

\subsection{Fluorimetry in solution}

Feasibility of fluorescence sensing in solution with the wireless instrument has been tested with a widely available fluorophore, fluorescein. Fluorescein has an absorption maximum at $491 \mathrm{~nm}$, so a cyan LED with a peak wavelength at $502 \mathrm{~nm}$ was found to be a good match. The fluorescence emission maximum is at $515 \mathrm{~nm}$ as can be seen from the spectra in $0.1 \mathrm{M}$ $\mathrm{NaOH}$, Fig. S2. The broadband photodiode used in these experiments is sensitive in the entire visible range, so the detected light was filtered with a bandpass optical filter placed across the photodiode, Fig 2b. Three different filters were tested: 530 - 900 nm, 550 - 900 $\mathrm{nm}$ and $570-900 \mathrm{~nm}$. It was found that filters $530-900 \mathrm{~nm}$ and $550-900 \mathrm{~nm}$ passed too much of the excitation light and so the low-level fluorescent light signal could not easily be detected. Due to the high intensity of the LED, the $570-900 \mathrm{~nm}$ filter was found to give best results and was therefore used for all subsequent experiments. However, this filter blocks a significant proportion of the fluorescence light at $515 \mathrm{~nm}$, along with the excitation light at $502 \mathrm{~nm}$. This was found to cause a slight issue of low sensitivity in the system, but may be improved and optimised in the future by using fluorophores with larger Stokes shift, LEDs with a shorter wavelength, narrowband photodiodes, different band pass filters, or indeed an optimised combination of all of the above.

In the first set of proof-of-concept experiments, different concentrations of the model analyte, fluorescein in $0.1 \mathrm{M} \mathrm{NaOH}$, were determined. The real-time fluorescence response is shown, Fig. 4a. At larger optical densities of the sample solution (i.e. larger concentrations of fluorescein), a pronounced inner filter effect is observed. The result of this is a deviation from the linear relationship between fluorescence intensity and concentration at higher concentrations, Fig. 4b. However, the response was found to be linear in the concentration range $10^{-7}$ to $10^{-5} \mathrm{M}$, with a linear response function determined with the wireless fluorimeter 
of $\mathrm{ADC}$ code $=7.913 \times 10^{7} \times c$ (fluorescein $)-336.1\left(\mathrm{R}^{2}=0.9959\right)$, Fig. 4c. In this relationship the ADC code represents fluorescence intensity in arbitrary units (a.u.). The limit of detection (LOD) of the wireless fluorimeter was calculated using the $\mu+3 \sigma$ methodology and was found to be $2.35 \times 10^{-7} \mathrm{M}$ fluorescein.
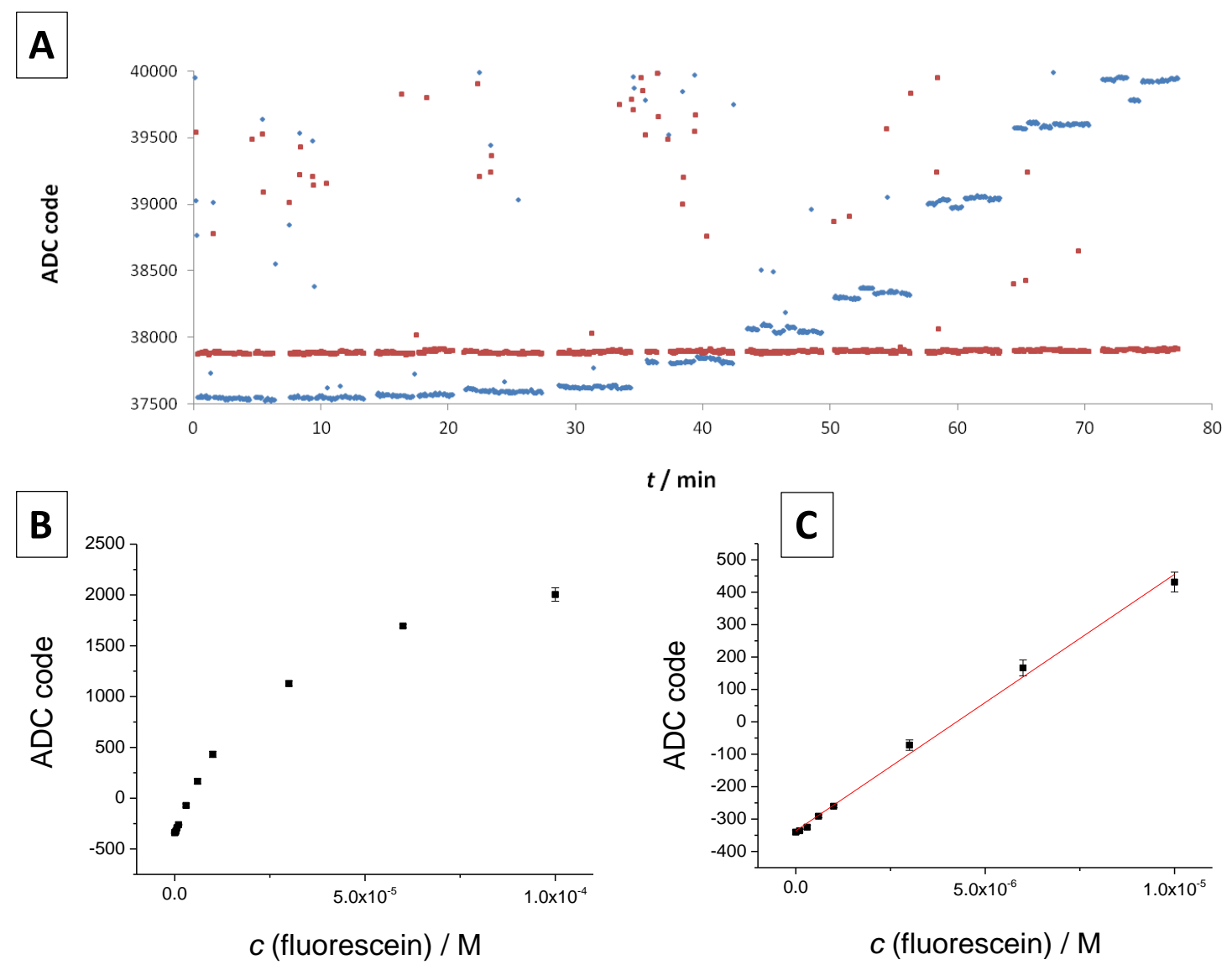

Figure 4. a) Real-time response of the wireless fluorimeter to increasing concentrations of fluorescein in solution (0 to $10^{-4} \mathrm{M}$ ) (blue points) and measured background light level (red points); b) Response showing the average measured fluorescence intensity (in arbitrary ADC code units) after subtraction of the background light level; c) The linear dependence of fluorescence intensity vs fluorescein concentration in the range $10^{-7}$ to $10^{-5} \mathrm{M}$.

\subsection{Paper-based chloride sensing}

The wireless fluorimeter was then used for chloride sensing with another common fluorophore, quinine sulphate, immobilised on filter paper test strips. The sensing mechanism is based on well-known fluorescence quenching of quinine by chloride ions [33, 
34]. Collisional quenching of fluorescence intensity by chloride is described by the SternVolmer equation:

$I_{0} / I=1+K_{\mathrm{sv}}\left[\mathrm{Cl}^{-}\right]$

where $I_{0}$ and $I$ are the fluorescence intensities in the absence and presence of chloride and $K_{\mathrm{sv}}$ is the Stern-Volmer quenching constant. Hence, from the decreased fluorescence intensity of the fluorophore, concentration of chloride in the sample can be calculated.

Since quinine sulphate has an excitation maximum at around $350 \mathrm{~nm}$ (Fig. S3), a UV LED with a peak wavelength at $355 \mathrm{~nm}$ was chosen for excitation. As the photodetector, a narrowband photodiode (EPD-470) was chosen, which is responsive in the range $385-565$ $\mathrm{nm}$, with a peak sensitivity at $470 \mathrm{~nm}$, and could effectively collect emitted fluorescence of QS $\left(\lambda_{\mathrm{em}, \max }=450 \mathrm{~nm}\right.$, Fig. S3). Another narrowband photodiode, which is responsive in the $410-580 \mathrm{~nm}$ range (EPD-525), was also tested but found to be less sensitive to the emitted QS fluorescence. By using this simple arrangement, all additional optical components (filters, lenses etc.) were eliminated from the setup. Furthermore, the UV LED light source and PD detector were placed next to each other in a planar configuration, Fig. 3b, which diversifies application possibilities of the wireless fluorimeter, especially for wearable sensing. Paper substrates are themselves an attractive platform for use in wearable sensors for sweat analysis, due to the possibility of passive sample manipulation by capillary forces [35]. The small volume of samples used in these experiments $(10 \mu \mathrm{L})$ also supports potential use of the system for sweat analysis.

The fluorescence intensity of QS-impregnated filter papers decreases upon exposure to increasing concentrations of chloride, as can be seen from the photograph in Fig 5a. The fluorescence intensity was recorded with the wireless fluorimeter in the range $1 \mathrm{mM}-100$ $\mathrm{mM}$ of $\mathrm{NaCl}$ and is shown in Fig 5b. Error bars represent one standard deviation around the mean, measured with 6 separate filter paper test strips. The relative standard deviation (RSD) was in all cases better than $9 \%$. When the initial fluorescence intensity $\left(I_{0}\right.$, measured with the addition of $10 \mu \mathrm{L}$ of water) is divided by the sample fluorescence intensity (I, measured with the addition of $10 \mu \mathrm{L}$ of $\mathrm{NaCl}$ sample) and plotted against chloride concentration, a characteristic linear Stern-Volmer relationship is obtained, Figure $5 \mathrm{c}$. We can see that a highly linear response $\left(R^{2}=0.9979\right)$ to chloride is obtained in the entire physiologically relevant concentration range [36]. Furthermore, a clear distinction can be made between $40 \mathrm{mM}$ and $60 \mathrm{mM}$ chloride solutions, which is necessary for potential 
application in cystic fibrosis diagnosis via sweat chloride assessment [37, 38]. The limit of detection, calculated using the $\mu+3 \sigma$ methodology, was found to be $1.11 \mathrm{mM}$ of chloride.
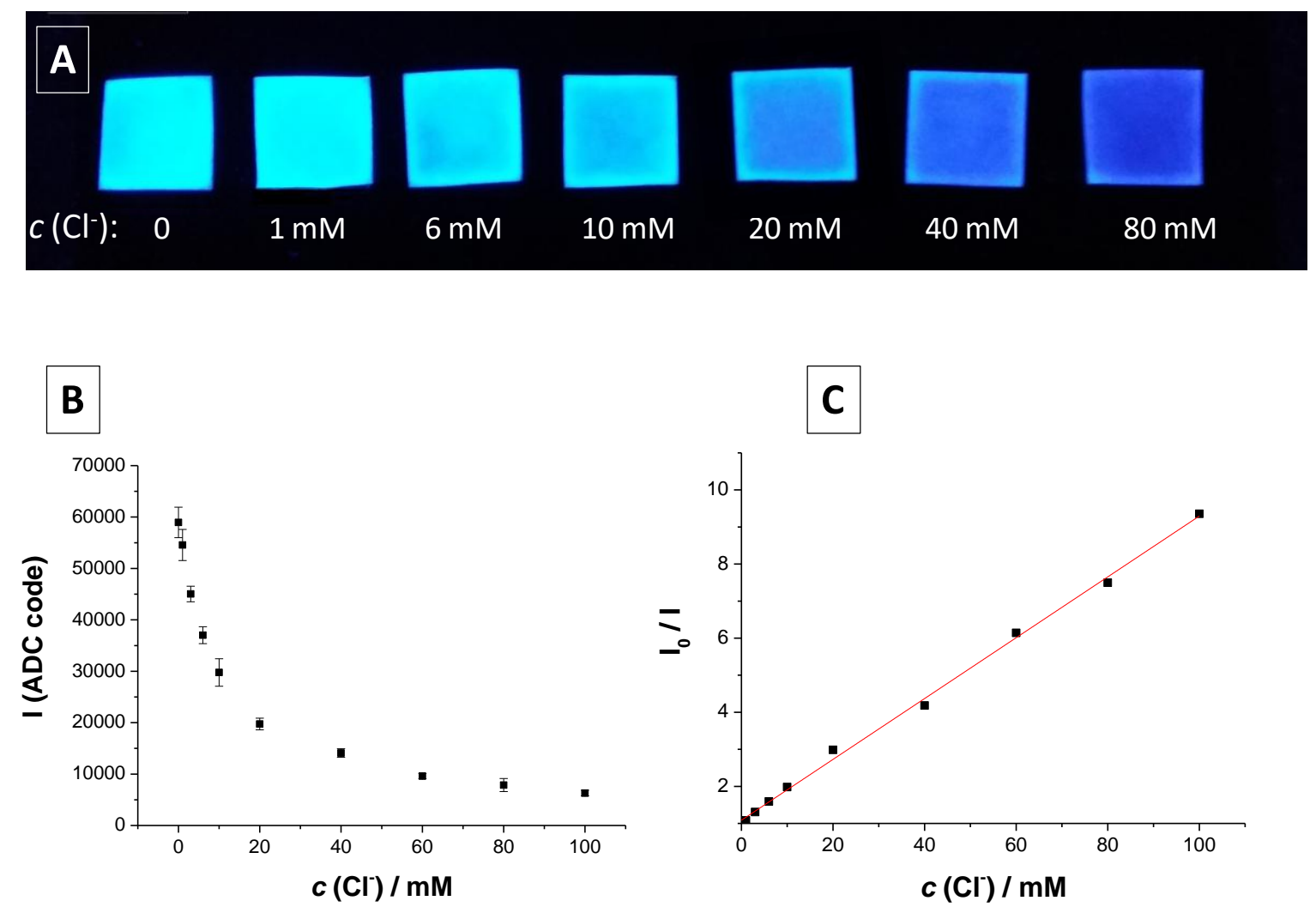

Figure 5. a) UV-illuminated photograph of quinine sulphate impregnated filter paper test strips upon exposure to increasing concentrations of chloride; b) Response of the wireless fluorimeter to increasing concentrations of chloride, error bars represent one standard deviation $(n=6)$; c) Stern-Volmer plot demonstrating a linear response in the entire physiologically relevant range of chloride concentration.

The presented system successfully overcomes some of the drawbacks of paper-based fluorescence sensing and could conveniently enable fluorescence detection in microfluidic paper based analytical devices ( $\mu$ PADs) [39]. Although this experimental setup allows only single-shot chloride tests, the proof-of-concept experiment demonstrates feasibility of using the RFID/NFC platform for continuous wireless fluorescence sensing with solid state luminescent films obtained by alternate immobilisation procedures [40]. Apart from chloride, the approach can be extended to sensing of other important sweat analytes, such as $\mathrm{Na}^{+}$, $\mathrm{K}^{+}, \mathrm{Ca}^{2+}$, with novel fluorescent sensors $[41,42]$. 


\section{Conclusion}

This work presents a low-cost and highly portable fluorimeter with RFID/NFC wireless connectivity, specifically aimed at mobile and wearable sensor applications. The analytical performance of the wireless fluorimeter has been demonstrated via two sets of proof-ofconcept fluorescence-based optical analytical experiments. Suitable analytical performance, in terms of precision, linearity and limit of detection, was achieved both in solution fluorimetry and solid-state chloride sensing with paper strips. The wireless fluorimeter has potential application with different mobile assay systems, where fluorescence intensity needs to be simply and rapidly determined at the point-of-sample. By changing out the optoelectronic components of the optical cell on individual daughter boards to match the desired indicator chemistry, a diverse range of fluorescent sensor chemistries can be implemented. With a wide palette of possible analytes, and the ability to modify the specific chemistry and optical measurement strategy, numerous mobile, wearable and near-to-point-of-sample applications for this type of system can be envisaged. With the expected explosion in the number of connected and wearable devices and the emergence of the Sensor Internet of Things, this wireless fluorimeter could provide an exciting analytical platform for ambient chemical intelligence.

\section{Acknowledgements}

These materials are based on work supported by the University of Zagreb, under research grant number 110011 (2013), 'Integrated analytical chemical systems: development and application of chemical sensors and biosensors.' The authors would like to thank Mario Popović of Dok-Ing d.o.o. for the 3D printing of the ABS optoelectronic probe holder. 


\section{References}

[1] R.A. Potyrailo, N. Nagraj, C. Surman, H. Boudries, H. Lai, J.M. Slocik, et al., Wireless sensors and sensor networks for homeland security applications, TrAC Trends in Analytical Chemistry, 40(2012) 133-45.

[2] D. Diamond, S. Coyle, S. Scarmagnani, J. Hayes, Wireless sensor networks and chemo/biosensing, Chemical Reviews, 108(2008) 652-79.

[3] P. Kassal, M.D. Steinberg, I.M. Steinberg, Wireless chemical sensors and biosensors: A review, Sensors and Actuators B: Chemical, 266(2018) 228-45.

[4] E. Strömmer, M. Hillukkala, A. Ylisaukko-oja, Ultra-low Power Sensors with Near Field Communication for Mobile Applications, in: L. Orozco-Barbosa, T. Olivares, R. Casado, A. Bermúdez (Eds.), Wireless Sensor and Actor Networks, Springer US2007, pp. 131-42.

[5] L. Atzori, A. Iera, G. Morabito, The Internet of Things: A survey, Computer Networks, 54(2010) 2787-805.

[6] D.Q. Zhang, Z.J. He, Y.M. Qian, J.F. Wan, D. Li, S.J. Zhao, REVISITING UNKNOWN RFID TAG IDENTIFICATION IN LARGE-SCALE INTERNET OF THINGS, Ieee Wireless Communications, 23(2016) 24-9.

[7] P. Kassal, Horak, E., Sigurnjak M., Steinberg, M.D., , I. Murković Steinberg, Wireless and Mobile Optical Chemical Sensors and Biosensors, Reviews in Analytical Chemistry, Under review, (2018).

[8] L.F. Capitán-Vallvey, A.J. Palma, Recent developments in handheld and portable optosensing--A review, Analytica Chimica Acta, 696(2011) 27-46.

[9] P.K. Dasgupta, I.-Y. Eom, K.J. Morris, J. Li, Light emitting diode-based detectors: Absorbance, fluorescence and spectroelectrochemical measurements in a planar flow-through cell, Analytica Chimica Acta, 500(2003) 337-64.

[10] M. O'Toole, D. Diamond, Absorbance Based Light Emitting Diode Optical Sensors and Sensing Devices, Sensors, 8(2008) 2453-79.

[11] M. Pokrzywnicka, L. Tymecki, R. Koncki, Low-cost optical detectors and flow systems for protein determination, Talanta, 96(2012) 121-6.

[12] X.-d. Wang, O.S. Wolfbeis, Fiber-Optic Chemical Sensors and Biosensors (2013-2015), Analytical Chemistry, 88(2016) 203-27.

[13] B. Valeur, I. Leray, Design principles of fluorescent molecular sensors for cation recognition, Coordination Chemistry Reviews, 205(2000) 3-40.

[14] M.D. Steinberg, P. Kassal, B. Tkalcec, I.M. Steinberg, Miniaturised wireless smart tag for optical chemical analysis applications, Talanta, 118(2014) 375-81.

[15] P. Kassal, M. Zubak, G. Scheipl, G.J. Mohr, M.D. Steinberg, I.M. Steinberg, Smart bandage with wireless connectivity for optical monitoring of $\mathrm{pH}$, Sensors and Actuators BChemical, 246(2017) 455-60.

[16] A. Martinez-Olmos, J. Fernandez-Salmeron, N. Lopez-Ruiz, A. Rivadeneyra Torres, L.F. Capitan-Vallvey, A.J. Palma, Screen Printed Flexible Radiofrequency Identification Tag for Oxygen Monitoring, Analytical Chemistry, 85(2013) 11098-105.

[17] P. Escobedo, M.M. Erenas, N. Lopez-Ruiz, M.A. Carvajal, S. Gonzalez-Chocano, I. de Orbe-Paya, et al., Flexible Passive near Field Communication Tag for Multigas Sensing, Analytical Chemistry, 89(2017) 1697-703.

[18] M. Mortellaro, A. DeHennis, Performance characterization of an abiotic and fluorescentbased continuous glucose monitoring system in patients with type 1 diabetes, Biosensors \& Bioelectronics, 61(2014) 227-31.

[19] A. Nemiroski, M. Ryou, C.C. Thompson, R.M. Westervelt, Swallowable fluorometric capsule for wireless triage of gastrointestinal bleeding, Lab on a Chip, 15(2015) 4479-87. 
[20] J.C. Contreras-Naranjo, Q. Wei, A. Ozcan, Mobile Phone-Based Microscopy, Sensing, and Diagnostics, Ieee Journal of Selected Topics in Quantum Electronics, 22(2016).

[21] A.J. Bandodkar, J. Wang, Non-invasive wearable electrochemical sensors: a review, Trends in Biotechnology, 32(2014) 363-71.

[22] G. Matzeu, L. Florea, D. Diamond, Advances in wearable chemical sensor design for monitoring biological fluids, Sensors and Actuators B-Chemical, 211(2015) 403-18.

[23] J. Heikenfeld, Non-invasive Analyte Access and Sensing through Eccrine Sweat: Challenges and Outlook circa 2016, Electroanalysis, 28(2016) 1242-9.

[24] M. Bariya, H.Y.Y. Nyein, A. Javey, Wearable sweat sensors, Nature Electronics, 1(2018) 160-71.

[25] M.D. Steinberg, P. Kassal, I.M. Steinberg, System Architectures in Wearable Electrochemical Sensors, Electroanalysis, 28(2016) 1149-69.

[26] P.M. Farrell, B.J. Rosenstein, T.B. White, F.J. Accurso, C. Castellani, G.R. Cutting, et al., Guidelines for diagnosis of cystic fibrosis in newborns through older adults: Cystic Fibrosis Foundation consensus report, Journal of Pediatrics, 153(2008) S4-S14.

[27] B.J. Rosenstein, G.R. Cutting, P. Cystic Fibrosis Fdn Consensus, The diagnosis of cystic fibrosis: A consensus statement, Journal of Pediatrics, 132(1998) 589-95.

[28] J. Gonzalo-Ruiz, R. Mas, C. de Haro, E. Cabruja, R. Camero, M.A. Alonso-Lomillo, et al., Early determination of cystic fibrosis by electrochemical chloride quantification in sweat, Biosensors and Bioelectronics, 24(2009) 1788-91.

[29] D.-H. Choi, Y. Li, G.R. Cutting, P.C. Searson, A wearable potentiometric sensor with integrated salt bridge for sweat chloride measurement, Sensors and Actuators B: Chemical, 250(2017) 673-8.

[30] A. Koh, D. Kang, Y. Xue, S. Lee, R.M. Pielak, J. Kim, et al., A soft, wearable microfluidic device for the capture, storage, and colorimetric sensing of sweat, Science Translational Medicine, 8(2016).

[31] J.P. Kim, Z. Xie, M. Creer, Z. Liu, J. Yang, Citrate-based fluorescent materials for lowcost chloride sensing in the diagnosis of cystic fibrosis, Chemical Science, 8(2017) 550-8.

[32] W. Junsheng, W. Xudong, C. Chanhee, G. Tanja, L. Dongqing, A novel device for quantitative measurement of chloride concentration by fluorescence indicator, Measurement Science and Technology, 23(2012) 025701.

[33] E. Urbano, H. Offenbacher, O.S. Wolfbeis, Optical sensor for continuous determination of halides, Analytical Chemistry, 56(1984) 427-9.

[34] A. Martin, R. Narayanaswamy, Studies on quenching of fluorescence of reagents in aqueous solution leading to an optical chloride-ion sensor, Sensors and Actuators B: Chemical, 39(1997) 330-3.

[35] S. Anastasova, B. Crewther, P. Bembnowicz, V. Curto, H.M.D. Ip, B. Rosa, et al., A wearable multisensing patch for continuous sweat monitoring, Biosensors and Bioelectronics, 93(2017) 139-45.

[36] Z. Sonner, E. Wilder, J. Heikenfeld, G. Kasting, F. Beyette, D. Swaile, et al., The microfluidics of the eccrine sweat gland, including biomarker partitioning, transport, and biosensing implications, Biomicrofluidics, 9(2015) 031301.

[37] J.L. Lezana, M.H. Vargas, J. Karam-Bechara, R.S. Aldana, M.E.Y. Furuya, Sweat conductivity and chloride titration for cystic fibrosis diagnosis in 3834 subjects, Journal of Cystic Fibrosis, 2(2003) 1-7.

[38] I. Sermet-Gaudelus, E. Girodon, D. Sands, N. Stremmler, V. Vavrova, E. Deneuville, et al., Clinical Phenotype and Genotype of Children with Borderline Sweat Test and Abnormal Nasal Epithelial Chloride Transport, American Journal of Respiratory and Critical Care Medicine, 182(2010) 929-36. 
[39] K. Yamada, H. Shibata, K. Suzuki, D. Citterio, Toward practical application of paperbased microfluidics for medical diagnostics: state-of-the-art and challenges, Lab on a Chip, 17(2017) 1206-49.

[40] W. Guan, W. Zhou, J. Lu, C. Lu, Luminescent films for chemo- and biosensing, Chemical Society Reviews, 44(2015) 6981-7009.

[41] K. Klucinska, E. Stelmach, A. Kisiel, K. Maksymiuk, A. Michalska, Nanoparticles of Fluorescent Conjugated Polymers: Novel Ion-Selective Optodes, Analytical Chemistry, 88(2016) 5644-8.

[42] A. Kisiel, K. Klucinska, M. Gniadek, K. Maksymiuk, A. Michalska, Optimizing calcium selective fluorimetric nanospheres, Talanta, 144(2015) 398-403. 


\title{
Supplementary Material
}

\section{Wireless Fluorimeter for Mobile and Low Cost Chemical Sensing: A Paper Based Chloride Assay}

\author{
Petar Kassal ${ }^{a}$, Matthew D. Steinberg ${ }^{b}$, Ema Horak ${ }^{c}$, Ivana Murković Steinberg ${ }^{a, \star}$
}

${ }^{a}$ Faculty of Chemical Engineering \& Technology, University of Zagreb, Marulićev trg 19, HR-10000 Zagreb, Croatia

${ }^{\mathrm{b}}$ GoSense Wireless Ltd, 57A Moorfield Road, Duxford, Cambridge, CB22 4PP, UK

${ }^{c}$ Computational Organic Chemistry and Biochemistry Group, Ruđer Bošković Institute, Bijenička cesta 54, HR-10000 Zagreb, Croatia

${ }^{*}$ Corresponding author:

Tel : +385 (0)1 4597287

E-mail: ivana.murkovic@fkit.hr

\subsection{Wireless fluorimeter design}

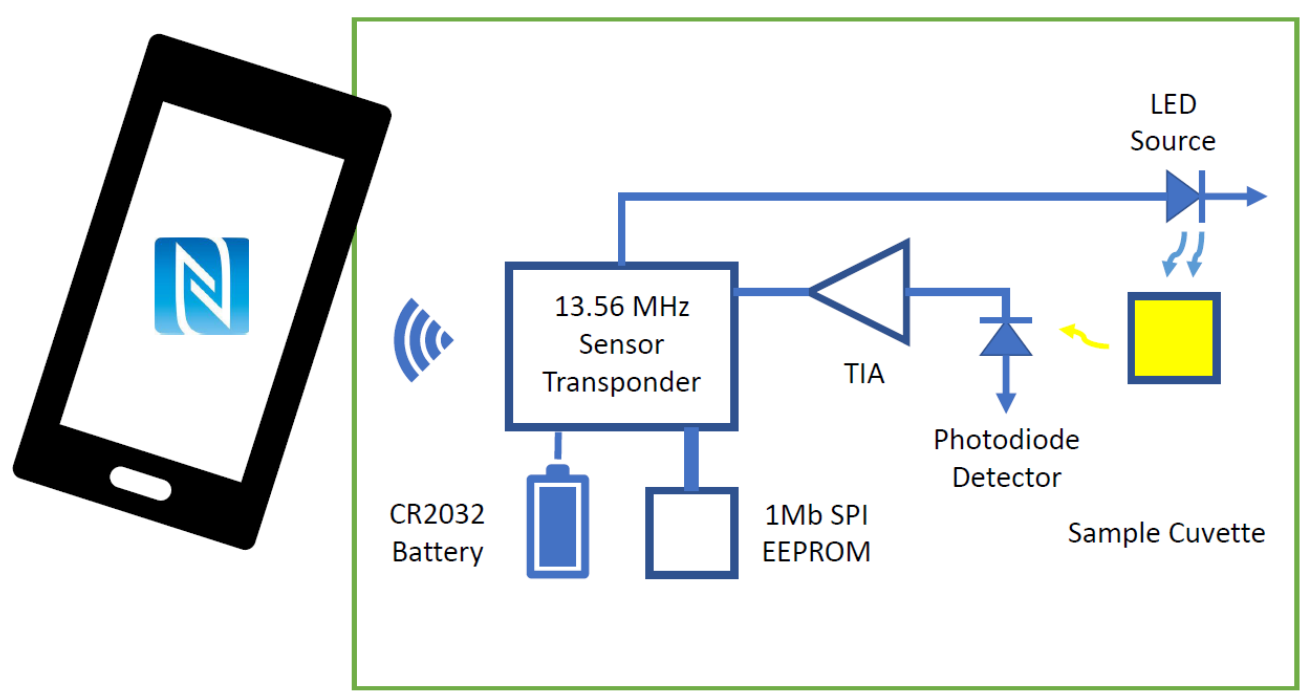

Figure S1 Schematic showing main components of the wireless fluorimeter and communication with NFC enabled smartphones. 
General protocol for fluorescence intensity measurements with the wireless fluorimeter

During a sample measurement, the LED turns on for $128 \mathrm{~ms}$ before any signals from the PD are measured. This provides an equilibration period. The LED is driven by a $20 \mathrm{~mA}$ constant current source. The analogue to digital converter then performs 32 successive 16-bit conversions of the amplified PD output, and averages them into one value before storing this single result to memory. This signal averaging provides excellent signal to noise ratio. The LED is then disabled and a background (reference) light level acquisition is made from the PD using the same method of averaging 32 sequential readings. One complete sample cycle (LED-on, equilibration, fluorescence measurement, LED-disabled, background measurement) takes $1472 \mathrm{~ms}$ to complete. This cycle can be programmed to occur at any time interval from once every $1.5 \mathrm{~s}$ to once in 10 days.

\subsection{Methods}

\subsubsection{Fluorimetry in solution}

Settings of the wireless fluorimeter in fluorimetry in solution experiments

The mobile fluorimeter was programmed with the following default settings prior to experimental use; $A D C$ mode $=11$-bit effective resolution, signal acquisition mode $=$ average of 32 samples, sensor initialisation time $=128 \mathrm{~ms}$, PD amplifier gain $=16$, data acquisition rate $=1$ sample every $5 \mathrm{~s}$. Cuvettes containing sample solutions were placed on the cell holder of the wireless fluorimeter, and optical data acquired for a period of one minute (12 recorded samples). During the acquisition period the system was in a dark enclosure. At the end of one minute of data acquisition the cuvette was removed from the fluorimeter, and the same cuvette then returned for a second minute of data sampling. The removal and return procedure was performed a total of 6 times, so that 6 minutes of data in total were acquired for each individual sample cuvette. After this, the solution was changed and the process repeated for the next sample, and so on until all samples had been processed in this manner. The last ten of each of the twelve data points sampled in each 1 minute period were kept, and the mean value calculated, thus giving six measurements per sample solution. The first two samples of each 1 minute period were rejected as these coincided with the placement and removal of the cuvettes into the fluorimeter. The mean and standard deviation about the mean were then calculated for these six 1-minute averages for each sample. 


\subsubsection{Paper-based chloride sensing}

Settings of the wireless fluorimeter in chloride sensing experiments

For the chloride sensing experiments, the mobile fluorimeter was programmed with the following default settings prior to experimental use; ADC mode $=11$-bit effective resolution, signal acquisition mode $=$ average of 32 samples, sensor initialisation time $=128 \mathrm{~ms}, \mathrm{PD}$ amplifier gain $=142$, data acquisition rate $=1$ sample every $2 \mathrm{~s}$. The first recorded point was taken as the fluorescence intensity (ADC code) value and used for subsequent calculations. This was repeated for a total of 6 times for each chloride solution, each time with a new QSimpregnated test strip. The recorded data was transferred wirelessly to a personal computer using the desktop RFID reader or to a smartphone running a custom-designed Android application via NFC.

\section{Results and discussion}

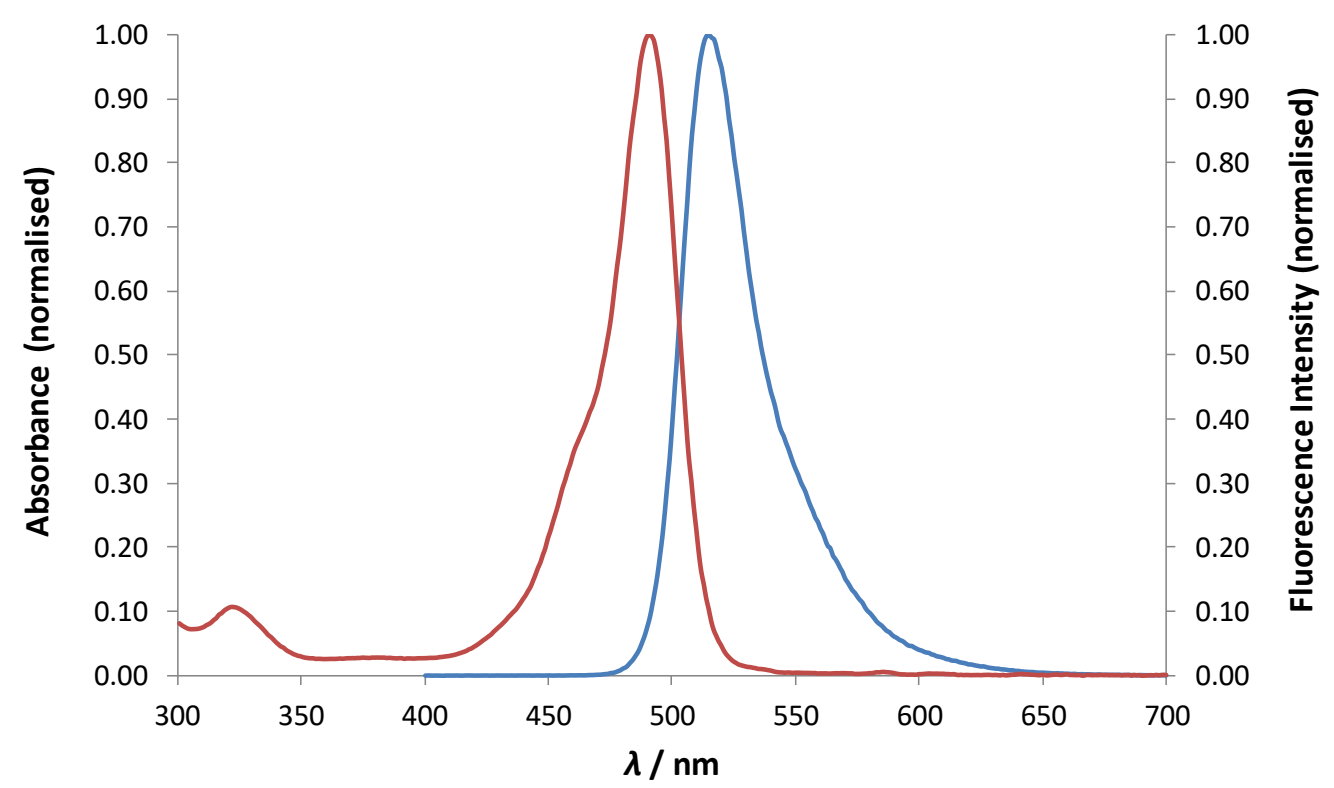

Figure S2 Normalised absorbance and fluorescence spectra of fluorescein in $0.1 \mathrm{M} \mathrm{NaOH}$. 


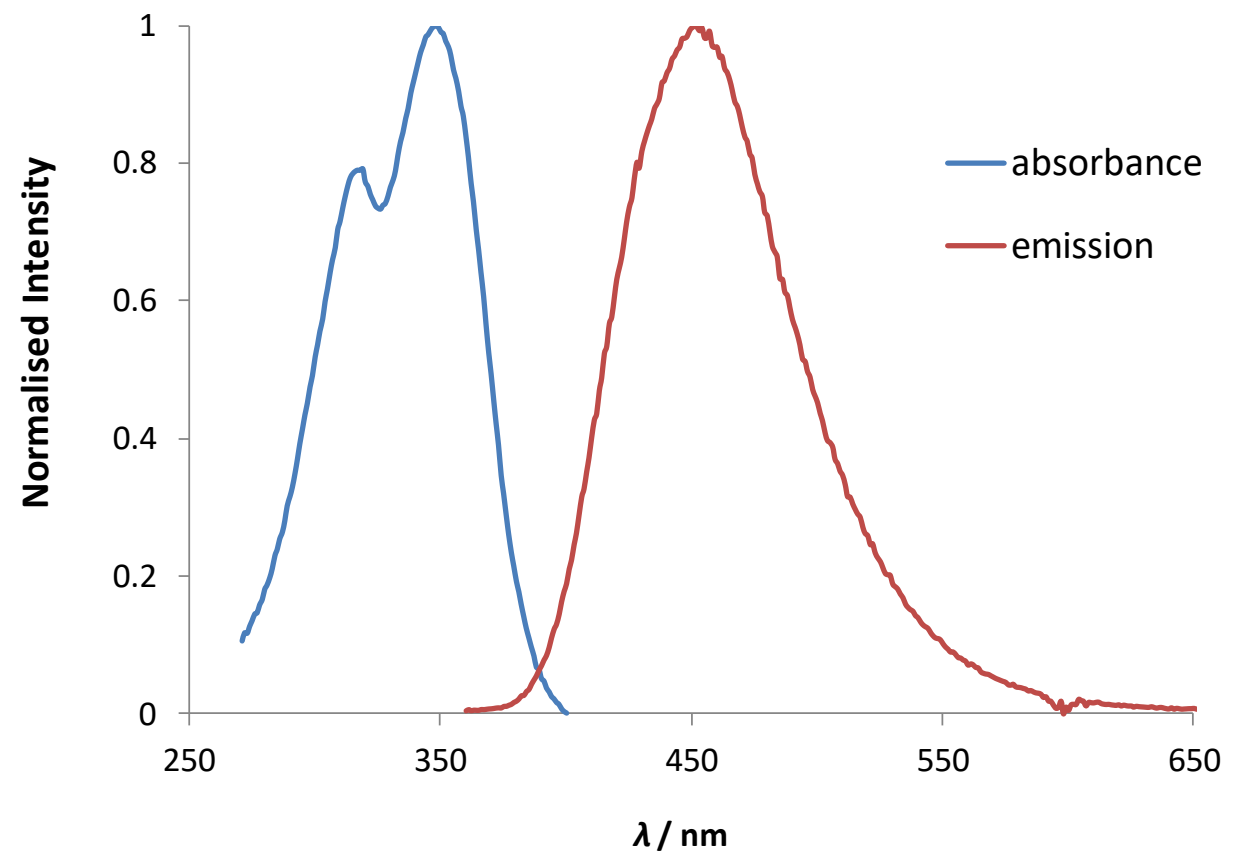

Figure S3 Normalised absorbance and fluorescence spectra of quinine sulphate in $0.5 \mathrm{M}$ $\mathrm{H}_{2} \mathrm{SO}_{4}$. 